López-Bedoya, J.; Vernetta-Santana, M.; Lizaur-Girón, P.: Martínez-Patiño, M.J.; Ariza-Vargas, L. (2020) Efficiency and Perception of Pain in Hold-Relax Stretching Techniques and Electrostimulation. Revista Internacional de Medicina y Ciencias de la Actividad Física y el Deporte vol. 20 (80) pp. 623-640 Http://cdeporte.rediris.es/revista/revista80/arteficacia1184.htm DOI: https://doi.org/10.15366/rimcafd2020.80.011

\title{
ORIGINAL
}

\section{EFICACIA Y PERCEPCIÓN DEL DOLOR EN TÉCNICAS DE ESTIRAMIENTOS HOLD-RELAX Y ELECTROESTIMULACIÓN}

\section{EFFICIENCY AND PERCEPTION OF PAIN IN HOLD- RELAX STRETCHING TECHNIQUES AND ELECTROSTIMULATION}

\author{
López-Bedoya, J.'; Vernetta-Santana, M. ${ }^{2}$; Lizaur-Girón, P. ${ }^{3}$ : Martínez- \\ Patiño, M.J. ${ }^{4}$ y Ariza-Vargas, L. ${ }^{5}$ \\ ${ }^{1}$ Departamento de Educación Física y Deportiva, Facultad de Ciencias de la Actividad Física y \\ del Deporte, Granada (España) jlopezb@ugr.es \\ 2 Departamento de Educación Física y Deportiva, Facultad de Ciencias de la Actividad Física y \\ del Deporte, Granada (España) vernetta@ugr.es \\ 3 Departamento de Educación Física y Deportiva, Facultad de Ciencias de la Actividad Física y \\ del Deporte, Universidad de Granada, Granada (España) plizaur@ugr.es \\ ${ }^{4}$ Departamento de Didácticas Especiales, Facultad de Ciencias de la Actividad Física y del \\ Deporte, Universidad de Vigo (España) mjpatino@uvigo.es \\ ${ }^{5}$ Departamento de Didáctica de la Expresión Artística y Corporal, Facultad de Ciencias de la \\ Educación, Universidad de Córdoba (España) eo1arval@uco.es
}

Código UNESCO / UNESCO Code: 241106 - Fisiología del ejercicio Clasificación Consejo de Europa / Council of Europe classification: 17. Otras (Entrenamiento deportivo) / Other (Sports training).

Recibido 3 de octubre de 2018 Received October 3, 2018

Aceptado 8 de noviembre de 2018 Accepted November 8, 2018

\section{RESUMEN}

Se analizó el efecto de la técnica de estiramiento HOLD RELAX sin (HR) y con electroestimulación (HR+EE) sobre la mejora y retención del rango de movimiento (ROM) activo (AROM) y pasivo (PROM) de cadera en flexión, y la percepción del dolor durante su aplicación. 42 deportistas fueron asignados a tres grupos: control, HR y HR+EE. El ROM fue medido con el test Straight-LegRaise antes, al finalizar el entrenamiento y trascurridas 2 semanas de su finalización. La valoración del dolor se realizó con la escala EVA. EI ANOVA mostró un aumento significativo del PROM $(p<0,001)$ en HR y HR+EE, no así del AROM. No se observaron pérdidas significativas del PROM en la retención de 
sendos grupos. En cuanto al dolor, no existieron diferencias significativas en los valores de EVA al aplicar ambas técnicas. Tanto HR como HR+EE fueron bien toleradas en cuanto a la percepción del dolor.

PALABRAS CLAVE: Flexibilidad, Entrenamiento, Electroestimulación, Extensibilidad isquiotibiales, Tolerancia al dolor.

\begin{abstract}
The objective of this study was to analyze the effect of the Hold-Relax stretching technique without (HR) and with electrostimulation (HR+EE) on the improvement and retention of active movement range (AROM) and passive (PROM) of hip in flexion, and the perception of pain during its application. 42 athletes were assigned to three groups: control, $\mathrm{HR}+\mathrm{EE}$ and $\mathrm{HR}$. The range of motion of the hip flexion was measured by test straight-leg-rise before, once completed and after 2 weeks of completion the training. Pain assessment was performed in all sessions with the EVA scale. The ANOVA showed a very significant increase in PROM $(p<0.001)$ in $H R$ and $H R+E E$, but not in AROM. No significant PROM losses were observed in the retention of both groups. Regarding pain, there were no significant differences in EVA values when applying both techniques. Both HR and HR+EE were well tolerated in terms of pain perception.
\end{abstract}

KEY WORDS: Training, Flexibility, Electrostimulation, Hamstring extensibility, Pain perception.

\title{
INTRODUCCIÓN
}

I papel la flexibilidad en el rendimiento deportivo ha sido ampliamente investigado con la esperanza de descubrir cómo las diferentes técnicas afectan a la mejora del rango de movimiento (ROM), tanto de forma aguda como crónica, ya que generalmente el incremento del ROM está asociado con una mejora en el rendimiento y una menor frecuencia de lesiones (Holt, Holt y Petham, 1996; Schmitt, Pelham y Holt, 1999). En deportes como la Natación Sincronizada, el Patinaje Artístico, especialidades gimnásticas como la Gimnasia Artística, Rítmica, Acrobática, Aeróbica, entre otras, requieren de importantes rangos de movimiento articular asociados a la mejora técnica y a la estética de los movimientos. El desarrollo de la flexibilidad en estas disciplinas, constituye un contenido importante del entrenamiento que se ve reflejado en el resultado de la competición. Es importante por tanto, entender los efectos de los distintos tipos de estiramiento y determinar cuándo es más apropiada cada técnica para maximizar el movimiento y el rendimiento humano (Bernhart, 2013).

Entre las tres técnicas básicas de estiramientos definidas por Page (2012), estáticas, dinámicas y estiramientos con pre-contracción, la Facilitación Neuromuscular Propioceptiva (FNP), situada dentro de los estiramientos con pre-contracción ha mostrado ser muy eficaz en la mejora del ROM activo y/o pasivo en diferentes articulaciones en deportistas (Zajac y Nowak, 1997; Kenric, 
2003; Funk, Swank, Mikla, Fagen y Farr, 2003; López-Bedoya, Vernetta, Robles y Ariza, 2013; García-Manso, López-Bedoya, Rodríguez-Matoso, Ariza-Vargas, Rodríguez-Ruiz y Vernetta, 2015).

Su eficacia se sustenta en aprovechar el reflejo de la inhibición autógena para conseguir inhibir la contracción muscular y así alcanzar mayores ROM. En definitiva, se trata de un método destinado a promover o acelerar la respuesta del mecanismo neuromuscular por medio de la estimulación de los propioceptores (Voss, lonta y Meyers, 2004).

Dentro de estas técnicas, son dos las más usadas relacionadas con el rendimiento físico: Contract-Relax (CR) y Hold-Relax (HR) (Surburg y Schrader, 1997; Adler, Berkers y Buck, 2002; Voss et al., 2004; López Bedoya et al., 2013). Su eficacia en la mejora del ROM activo y pasivo a largo plazo está suficientemente contrastada (Zajac y Nowak, 1997). Sin embargo, existe poca evidencia de su efecto cuando se aplica electroestimulación para ver la mejora sobre el ROM (Pérez y Álamo, 2001; Espejo, Maya, Cardero y Albornoz, 2012; López-Bedoya, Vernetta, Lizaur, Martinez-Patiño y Ariza-Vargas, 2017).

Por otro lado, uno de los factores que a menudo se presenta en el entrenamiento de la flexibilidad en general, es el dolor, consecuencia de una elongación mantenida de los tejidos.

La International Association for Study of Pain, define el dolor como una experiencia subjetiva, sensorial y emocional desagradable con un daño tisular potencial o real o descrito en términos de ese daño (Merskey y Bogduk, 2005). Los nociceptores son receptores periféricos del dolor que envían mensajes por medio de neurotransmisores a la vía central, siendo su función mantener la integridad del cuerpo y desencadenar respuestas (somáticas o vegetativas) asociadas a sensaciones dolorosas que tratan de diferenciar los estímulos inocuos de los lesivos. Esta función la realizan ignorando los estímulos de baja intensidad y codificando el estímulo lesivo dentro de un rango de intensidades y transmitiéndolo al SNC (Besson y Chaouch, 1987).

No se conocen con exactitud los mecanismos que sustentan el cambio en la percepción o tolerancia del estiramiento, solamente algunos autores han sugerido y estudiado la modulación del dolor en los estiramientos (Marshall, Cashman y Cheema, 2006; Sharman, Cresswel y Riek, 2006). Esta escasez de estudios hace interesante conocer la percepción del dolor durante el estiramiento muscular en todas las sesiones de entrenamiento, en tanto que puede presentarse como un factor limitante de dicho proceso. También Sands, Mc Neal, Stone, Haff y Kinser (2008) comprobaron en un estudio agudo de estiramiento mediante técnicas vibratorias y no vibratorias que no aparecieron diferencias entre ellas en la percepción del dolor a la presión post estiramiento medida por algometría. 


\section{OBJETIVOS}

El objetivo principal del estudio fue evaluar el efecto a largo plazo del entrenamiento de la flexibilidad mediante la técnica de FNP Hold-Relax sin (HR) y con la aplicación de electroestimulación (HR+EE), sobre el rango de movimiento pasivo y activo de la musculatura isquiotibial en practicantes de gimnasia no competitiva. Igualmente, se pretende conocer cuál de los dos tipos entrenamientos permite mantener la mejora obtenida, tras un periodo de inactividad de dos semanas, así como, analizar la percepción subjetiva de "dolor" de los sujetos en los tipos de estiramiento.

\section{MATERIAL Y MÉTODO}

\section{Participantes}

Un total de 42 jóvenes deportistas (22 hombres y 20 mujeres), estudiantes universitarios en Ciencias del Deporte, especialidad Gimnasia Artística (edad, $22,95 \pm 2,03$ años; masa corporal, 67,74 \pm 9,60 kg; talla, 171,63 \pm 9,37 cm), fueron asignados a 3 grupos: control, Hold-Relax inducida a través de electroestimulación y sin electroestimulación, mediante técnicas de bloqueo en función de los datos obtenidos en el pre-test. Ninguno de ellos padecía lesiones en la musculatura indicada, ni ninguna otra dolencia. Todos los participantes fueron plenamente informados de los procedimientos y posibles riesgos antes de obtener su consentimiento por escrito. El estudio fue aprobado por el Comité de Ética de la Universidad de Granada.

\section{Diseño experimental}

Se utilizó un diseño factorial de medidas repetidas, con tres niveles en el factor inter-grupo (control, HR+EE, HR) y tres niveles en el factor intra-grupo (medida pre-, post- y re-test). Las variables dependientes fueron el rango de movimiento pasivo (PROM) y el rango de movimiento activo (AROM) de la flexión de cadera de pierna dominante, así como, la percepción subjetiva del dolor por parte de los deportistas del estudio.

\section{Instrumentación y equipo}

Se utilizó una cámara fotográfica digital estándar (Nikon, Coolpix S500, Nikon Corporation, Chiyoda-ku, Tokio, Japón, http://www.nikon.com/) para capturar la imagen durante las pruebas de evaluación con el test Straight Leg Raise (SLR), con elevación activa y pasiva de la extremidad inferior en extensión (ASLR y PSLR, respectivamente) y posteriormente recoger los datos de los ángulos. Por otro lado, en la fase de entrenamiento se contó con 7 aparatos de electroestimulación programables, marca Cefar Myo4, con una forma de impulso rectangular bifásica asimétrica, una intensidad de los impulsos entre 0 y $120 \mathrm{~mA}$, en cada uno de sus cuatro canales y una anchura del impulso o duración calculada en microsegundos ( $\mu \mathrm{s})$. La frecuencia del impulso es programable en un rango comprendido entre 0 y $120 \mathrm{~Hz}$. Para la medición de la percepción del dolor se usó la escala analógica digital (EVA). 


\section{Procedimiento de medición}

Los test de evaluación se realizaron en tres momentos: el pre-test se realizó en una sesión previa a la primera sesión de entrenamiento; el post-test inmediatamente después de la última sesión de entrenamiento y el re-test, tras dos semanas de inactividad. Una vez realizada la evaluación del ASLR y PSLR respectivamente, los ángulos se midieron a través de la digitalización de los puntos anatómicos con el software ATD 2.0 para Windows (Analysis of Sport Techniques, Universidad de Granada, España), utilizando las fotografías tomadas durante los test de evaluación. El ángulo (a) se obtuvo digitalizando 3 puntos: el tobillo (maléolo), la cadera (trocánter mayor) y el tobillo de la otra extremidad inferior (maléolo). Cada fotografía fue digitalizada 3 veces en ASLR y PSLR, considerando para el análisis estadístico el valor promedio en cada caso. Durante los test, los participantes permanecieron tendidos sobre un banco, indicándoles que mantuvieran la cabeza alineada con la espalda, y presionando la zona lumbar contra el banco durante todo el ensayo. Manteniendo las rodillas completamente extendidas, cada participante procedió a levantar lentamente la extremidad inferior hacia arriba, flexionando la cadera evitando rotaciones internas y externas de la extremidad o desviaciones del plano sagital. Cuando se alcanzó la ROM máxima, activa o pasiva (según proceda), indicada oralmente por el participante, se mantuvo la posición y se tomó una fotografía con la cámara digital perpendicular al participante a una distancia de 4 metros, con el centro de enfoque en la articulación de la cadera.

La intensidad del dolor y su componente de disconfort han sido evaluados por diversos métodos, aunque tradicionalmente se han utilizado escalas numéricas o verbales para cuantificar el dolor, siendo la escala visual analógica (EVA) la más usada en los últimos 15 años (Olesen, Andresen, Staahl y Drewes, 2012).

En nuestro estudio hemos optado por la escala EVA, y la percepción del dolor se realizó inmediatamente después de cada sesión de estiramiento. Los participantes debían marcar en la planilla EVA la percepción de su dolor en una escala numérica de 0 a 10 puntos, delimitados por el extremo izquierdo "sin dolor" y derecho "peor dolor". Los participantes marcaban una línea vertical a través de la línea horizontal en el punto que mejor representara la intensidad máxima del dolor experimentada durante la sesión de estiramiento.

\section{Protocolo de entrenamiento}

Los participantes de los dos grupos experimentales realizaron un entrenamiento de flexibilidad de 2 sesiones por semana, durante 4 semanas. Antes de cada sesión de entrenamiento se realizó un calentamiento estandarizado durante 10 minutos, manteniéndose el mismo para todas las sesiones de entrenamiento y para ambos grupos. Las pruebas de evaluación en el pre-test, post-test y re-test y las sesiones de entrenamiento tuvieron lugar a la misma hora y en el mismo lugar, con una temperatura ambiental de $23^{\circ}$. El ejercicio de estiramiento utilizado fue la elevación de la extremidad inferior extendida (SLR, Straight-Leg- 
Raise). La sesión se utilizó como primer contacto y familiarización con la prueba de evaluación y la técnica empleada.

Grupo experimental 1 (HR+EE) (14 participantes): a este grupo se le aplicó la técnica HR con electroestimulación, consistente en una serie de 10 repeticiones del siguiente ciclo: elongación pasiva (PE) del grupo de músculos isquiotibiales hasta alcanzar el máximo ROM; contracción concéntrica isométrica (IC) del músculo a estirar (isquiotibiales) con electroestimulación. Para provocar dicha contracción se aplicó una corriente eléctrica bipolar (100 mA), a través de dos electrodos de superficie ubicados en los extremos proximal y distal de la musculatura isquiotibial. Los parámetros de corriente utilizados fueron los siguientes: Frecuencia del impulso eléctrico (FI) de $80 \mathrm{~Hz}$, con series de un tiempo de contracción (TC) de $6 \mathrm{~s}$; relajación de la contracción durante 2 s; 10 segundos de estiramiento pasivo asistido de la musculatura isquiotibial y $2 \mathrm{~s}$. de relajación de la musculatura en la posición inicial, resultando un tiempo total de estiramiento de $100 \mathrm{~s}$ por sesión y un tiempo de trabajo total de $3 \mathrm{~min}$. El grupo experimental 2 (HR), (14 participantes): la técnica aplicada fue la misma, si bien, la contracción isométrica se producía de forma voluntaria y una oposición controlada por un ayudante, en este grupo no se utilizó electroestimulación. Por otra parte un grupo de control (14 participantes) no realizaría ningún tipo de estiramiento.

\section{Análisis estadístico}

Previamente a su análisis, se comprobaron los supuestos de normalidad y homocedasticidad mediante los estadísticos de Shapiro Wilk y Levene, respectivamente.

Se analizaron las diferencias observadas en cada una de las variables dependientes AROM y PROM mediante un ANOVA factorial mixto o 'split-plot' (Tratamiento x Medida), con tres niveles en el factor inter-sujetos (control, HR+EE y HR) y tres niveles en el factor intra-sujetos (pre-, post- y re-test).

Se comprobó la igualdad de las matrices de varianzas y covarianzas de los niveles del factor intra-sujeto en cada uno de los niveles de los factores intersujeto mediante la prueba de esfericidad de Mauchly, utilizándose el estadístico F univariado, aplicando la estimación Greenhouse-Geisser del índice corrector Épsilon en caso de incumplimiento del supuesto de esfericidad.

En las comparaciones múltiples referidas a los efectos intra-sujetos se ajustaron los niveles críticos y los intervalos de confianza mediante la corrección de Bonferroni. El estudio del dolor con la EVA se llevó a cabo mediante un diseño Split-plot, considerando dos niveles (HR+EE y HR) del factor inter-sujetos Tratamiento, y un factor intra-sujetos Medida, con ocho niveles. La fiabilidad de las tres digitalizaciones de cada fotografía se abordó mediante el coeficiente de correlación intraclase (ICC). El nivel de significación usado en todos los test fue $\mathrm{p}<, 05$. 


\section{RESULTADOS}

Se comprobó la ausencia de diferencias significativas entre los valores medios de los registros en cada una de las variables dependientes en la medida Pretratamiento, mediante un ANOVA.

Todas las distribuciones de los valores correspondientes a las subpoblaciones resultantes de combinar los distintos niveles del factor Medida y Tratamiento mostraron un comportamiento normal, $\mathrm{p}<, 05$.

En la Tabla 1 se muestran los valores medios y desviaciones estándar de las distintas medidas realizadas de AROM y PROM y la existencia o no de diferencias significativas.

Tabla 1. Valores medios (y desviación estándar) de los rangos de movimiento de flexión de cadera, activo y pasivo, medidos en grados, según los distintos grupos experimentales y niveles del factor Medida (pre-, post- y retest).

\begin{tabular}{llrrrr}
\hline & Medida & \multicolumn{1}{c}{ Control } & \multicolumn{1}{c}{ HR } & \multicolumn{1}{c}{ HR+EE } & \multicolumn{1}{c}{ Subtotal } \\
\hline AROM & Pre- & $94,99(14,35)$ & $97,28(15,12)$ & $93,83(9,68)$ & $95,36(13,02)$ \\
& Post- & $93,46(13,69)$ & $99,97(13,90)$ & $97,34(10,64)$ & $96,92(12,80)$ \\
& Re-test & $93,81(16,49)$ & $97,89(14,88)$ & $95,79(10,99)$ & $95,83(14,06)$ \\
& Subtotal & $94,09(14,54)$ & $98,38(14,33)$ & $95,65(10,30)$ & \\
\hline PROM & Pre- & $123,60(17,07)$ & $127,78(20,27)$ & $129,04(21,80)$ & $126,81(19,46)$ \\
& Post- & $122,55(18,55)$ & $137,55(21,65)^{\dagger+}$ & $142,57(12,83)^{\star \dagger \dagger}$ & $134,22(19,59)^{\dagger+}$ \\
& Re-test & $127,86(19,85)$ & $137,58(19,34)^{\dagger+}$ & $138,08(12,62)^{\ddagger \ddagger}$ & $134,51(17,80)^{\dagger \dagger}$ \\
& Subtotal & $124,67(18,21)$ & $134,30(20,47)$ & $136,56(16,91)$ & \\
\hline
\end{tabular}

AROM (Rango Activo de Movimiento), PROM (Rango Pasivo de Movimiento)

HR (Facilitación Neuromuscular Propioceptiva - Hold Relax), HR+EE (Facilitación Neuromuscular Propioceptiva - Hold Relax con contracción isométrica inducida mediante electroestimulación)

${ }^{*}=p<.05$; diferencias estadísticamente significativas con Grupo Control

$\dagger \dagger=p<.001$; diferencias estadísticamente significativas con medida Pre-tratamiento

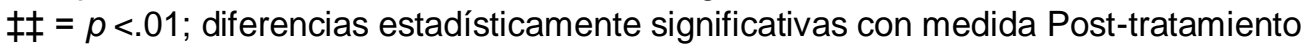

\section{Análisis del efecto de la aplicación HR+EE y HR sobre el ROM Activo y Pasivo}

El análisis factorial mixto arrojó resultados desiguales, en el rango de movimiento pasivo y activo. EI AROM no mostró diferencias significativas considerando las tres mediciones realizadas $F(2,78)=1,499, p=, 230, \eta_{p}^{2}=, 037$, ni atendiendo al efecto de la interacción de las variables Medida * Tratamiento $F(4,78)=1,482$, $p=, 216, \eta_{p}^{2}=, 071$. Tampoco se detectaron diferencias inter-sujetos según los distintos niveles del Tratamiento $F(2,39)=, 389, p=, 680, \eta_{p}^{2}=, 020$ (véase Figura $1 \mathrm{~A})$.

En PROM se registró un efecto principal significativo de la variable Medida $F(2$, $78)=15,457, p=, 000, \eta_{p}^{2}=, 284$ y de la interacción Medida * Tratamiento $F(4$, $78)=4,068, p=, 005, \eta_{p}^{2}=, 173$ ), (Figura 1B). El valor medio en la medida Pre$(M=126,81, S D=19,46)$, fue significativamente inferior $(p<, 001$ en ambos casos) a los valores medios registrados tras la aplicación del tratamiento $(M=$ $134.22, S D=19.59)$ y tras dos semanas de finalizar el tratamiento $(M=134,51$, 
$S D=17,80)$. Respecto al efecto de la interacción Medida * Tratamiento, el contraste univariado arrojó diferencias significativas en la medida post-test, $F(2$, $39)=4,663, p=, 015, \eta_{p}^{2}=, 193$, localizando las mismas entre el grupo Control $(M=122,55, S D=18,55)$ y el grupo tratado con $H R+E E(M=142,57, S D=$ $12,83), p=, 017,95 \% \mathrm{Cl}(2,954-37,084)$.
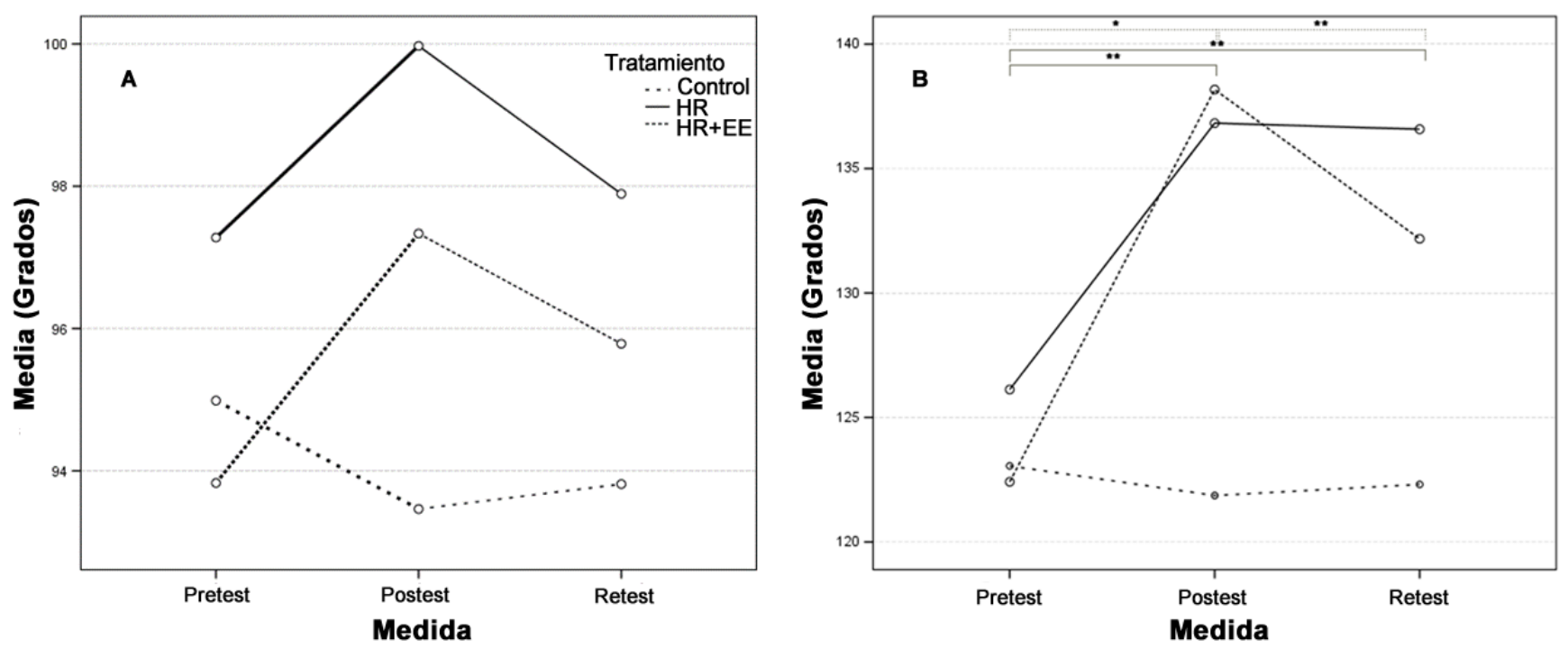

Figura 1. Valor medio del rango de movimiento activo $(A)$ y pasivo $(B)$ en cadera de extremidad inferior dominante, según los diferentes grupos experimentales (Control, HR y HR + EE) y las diferentes medidas.

$$
{ }^{*}=\mathrm{p}<, 001 ;{ }^{* *} \mathrm{p}<, 05
$$

El ANOVA de medidas repetidas sobre los tres niveles de la variable Medida, en cada uno de los niveles del factor Tratamiento, arrojó diferencias significativas en HR, $F(2,26)=13,191, p=, 000, \eta_{p}^{2}=, 504$, y HR+EE, $F(2,26)=6,27, p=$ $, 006, \eta_{p}{ }^{2}=, 325$ (Figura 2). En el primer caso, el valor medio de PROM en la medida pre-tratamiento $(M=127,78, S D=20,27)$ fue significativamente inferior al registrado en las medidas post-tratamiento $(M=137,55, S D=21,65), p=, 006$, $95 \% \mathrm{Cl}(2,839,16,691)$, y re-test $(M=137,58, S D=19,34), p=, 001,95 \% \mathrm{Cl}$ $(4,512,15,081)$. En el caso de HR+EE, estas diferencias se localizaron entre los valores medios de los registros pre- $(M=129,04, S D=21,80)$ y post-test $(M=$ $142,57, S D=12,83), p=, 032,95 \% \mathrm{Cl}(1,06,25,997)$, y entre éste último y la medida re-test $(M=142,57, S D=12,83), p=, 004,95 \% \mathrm{Cl}(1,458-7,512)$ (Tabla 1).

No se observaron diferencias entre los valores medios registrados en el factor inter-grupo Tratamiento: $F(2,39)=1,815, p=, 176, \eta_{p}^{2}=, 085$. 


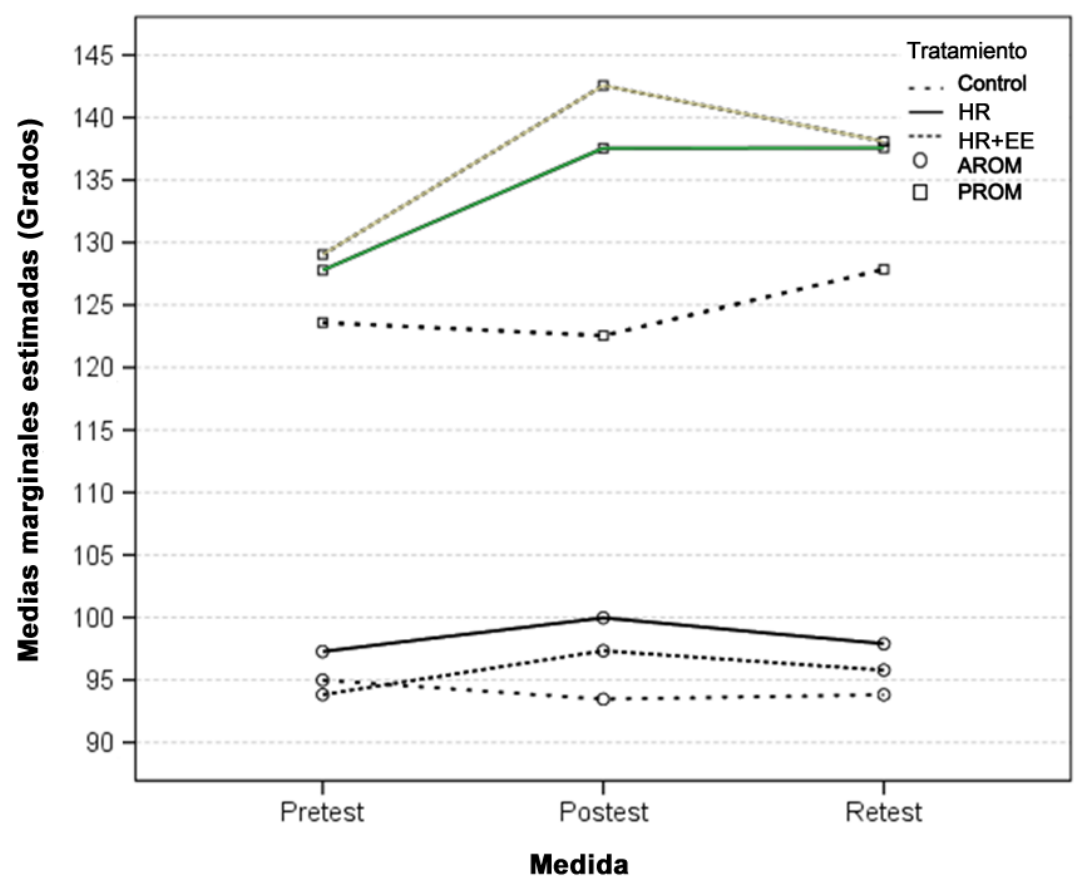

Figura 2. Valores medios de los rangos de movimiento, activo y pasivo, en cadera de extremidad inferior dominante, según los grupos experimentales (Control, HR y HR+EE) y medidas registradas.

\section{Análisis estadístico de EVA.}

Las tablas 2 y 3 recogen las pruebas de normalidad mediante el estadístico de Shapiro Wilk y los valores promedio y desviación estándar respectivamente. El ANOVA de medidas repetidas indicó la ausencia de efectos fijos estadísticamente significativos del factor Medida, $F(7,108,288)=, 924, p=, 455$, $\eta_{p}^{2}=, 034$. Por el contrario, se observaron diferencias estadísticamente significativas en las interacción Medida x Grupo, $F(7,108,288)=3,091, p=, 017$, $\eta_{p}{ }^{2}=, 106$. En concreto, el valor promedio de EVA fue estadísticamente diferente entre los tratamientos HR y HR+EE en la medida $1(M=57,66, S D=13,30$ y $M$ $=38,3, S D=17,29$, para HR y HR+EE respectivamente) $p=, 003 ; 95 \% \mathrm{IC}(7,374$ $-31,341)$. Únicamente en el grupo sometido a HR+EE se registraron diferencias significativas entre pares de medidas, en el emparejamientos $1(M=38,30, S D$ $=17,29)-4(M=57,73, S D=16,10), p=, 035 ; 95 \%$ IC $(0,7742-38,083)$ (Figura 4). Respecto al factor inter-sujeto, no se observaron diferencias estadísticamente significativas según el método aplicado (HR vs. $\mathrm{HR}+\mathrm{EE}), F(1,26)=, 232, p=$ ,634, $\eta_{p}^{2}=, 009$ (Figura 3). 
Rev.int.med.cienc.act.fís.deporte - vol. 20 - número 80 - ISSN: 1577-0354

Tabla 2. Prueba de normalidad de Shapiro Wilk, para cada medida registrada en cadera de extremidad inferior dominante.

\begin{tabular}{crrr}
\hline Medida & $\begin{array}{c}\text { Prueba } \\
\text { estadística }\end{array}$ & Gl & \multicolumn{2}{l}{ Sig. } \\
\hline 1 &, 974 & 28 &, 679 \\
2 &, 958 & 28 &, 306 \\
3 &, 971 & 28 &, 604 \\
4 &, 971 & 28 &, 599 \\
5 &, 975 & 28 &, 732 \\
6 &, 979 & 28 &, 826 \\
7 &, 969 & 28 &, 542 \\
8 &, 983 & 28 &, 906 \\
\hline
\end{tabular}

Tabla 3. Análisis descriptivo (media y desviación estándar) de EVA, en extremidad inferior dominante, según los distintos grupos experimentales.

\begin{tabular}{|c|c|c|c|c|c|c|c|c|c|}
\hline \multirow{2}{*}{ Medida } & \multicolumn{3}{|c|}{$\mathrm{HR}$} & \multicolumn{3}{|c|}{$\mathrm{HR}+\mathrm{EE}$} & \multicolumn{3}{|c|}{ TOTAL } \\
\hline & $n$ & $M$ & $S D$ & $N$ & $M$ & $S D$ & $n$ & $M$ & $S D$ \\
\hline 1 & 14 & 57,66 & 13,30 & 14 & 38,30 & 17,29 & 28 & 47,98 & 18,06 \\
\hline 2 & 14 & 50,38 & 14,20 & 14 & 48,32 & 11,76 & 28 & 49,35 & 12,83 \\
\hline 3 & 14 & 55,12 & 19,01 & 14 & 52,98 & 16,58 & 28 & 54,05 & 17,54 \\
\hline 4 & 14 & 48,33 & 19,08 & 14 & 57,73 & 16,11 & 28 & 53,03 & 17,97 \\
\hline 5 & 14 & 52,07 & 16,31 & 14 & 53,86 & 19,85 & 28 & 52,96 & 17,85 \\
\hline 6 & 14 & 52,07 & 17,91 & 14 & 51,93 & 21,36 & 28 & 52,00 & 19,35 \\
\hline 7 & 14 & 53,54 & 19,34 & 14 & 52,00 & 22,83 & 28 & 52,77 & 20,77 \\
\hline 8 & 14 & 57,07 & 16,18 & 14 & 51,21 & 20,19 & 28 & 54,14 & 18,20 \\
\hline
\end{tabular}




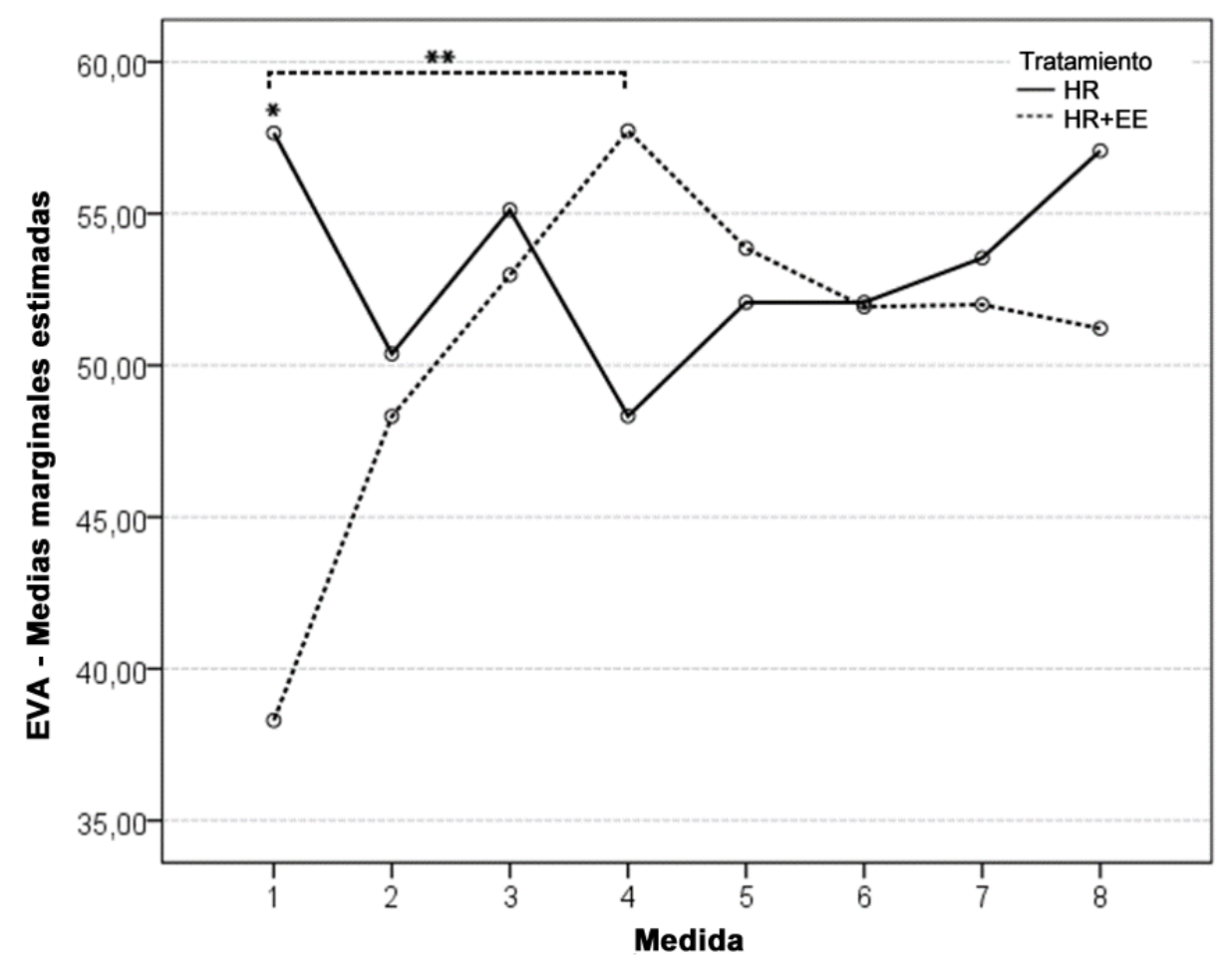

Figura 3. Valores medios de EVA en las ocho medidas (extremidad inferior dominante), según los diferentes grupos experimentales.

$\mathrm{HR}=$ Facilitación Neuromuscular Propioceptiva - Hold Relax; HR+EE = Facilitación Neuromuscular Propioceptiva - Hold Relax con contracción isométrica inducida mediante electroestimulación.

$$
{ }^{*}=p<.05 ;{ }^{* *}=p<.01
$$

\section{DISCUSION}

\section{Cambios producidos en el ROM pasivo y activo entre el pre y pos-test}

En el PROM, el ANOVA mostró diferencias estadísticamente significativas, con $p<, 01$ para la variable "medias-pasivas" (PROM) en las dos técnicas de entrenamiento HR y HR+EE entre las medidas pre y post-test. Los dos grupos experimentales tienen mejoras significativas del PROM $(p<, 001)$ tras el entrenamiento. Estudios similares han obtenido mejoras significativas utilizando técnicas de HR con dos sesiones de entrenamiento semanal, pero con un periodo mayor (Rowlands, Marginson y Lee, 2003; González-Ravé, SánchezGómez y Sánchez-García, 2012; López-Bedoya et al., 2013). López-Bedoya, Robles, Vernetta, Piedra y Núñez (2007), al comparar técnicas de HR y Active Isolated Stretching (AIS), evaluando el PROM en abducción, concluyeron que ambas técnicas fueron efectivas sin diferencias entre ellas. En un estudio posterior, López-Bedoya et al. (2013), comparando las mismas técnicas en isquiotibiales obtuvieron ganancias mayores con un PROM de 18,42ำ para AIS, y $17,18^{\circ}$ para HR, frente a $10,44^{\circ}$ para AIS y $6,52^{\circ}$ al aplicar HR en aductores. Con las mismas sesiones, tiempo y protocolo, el entrenamiento es más efectivo en la musculatura isquiotibial que en los músculos aductores, de manera que la ganancia de PROM puede estar relacionada con la técnica utilizada y también con la arquitectura muscular implicada. En nuestro estudio, la técnica de HR, obtuvo mejora significativa $(p<, 001)$ entre pre y post-test, con una ganancia de 
$9,77^{\circ}$ en el PROM, y mejoras significativas $(p<, 001)$, entre las medidas pre- $y$ post-test con la técnica HR+EE, y una ganancia de 13,53‥ Esto confirma la eficacia de esta técnica para la mejora del PROM como en el estudio de LópezBedoya et al. (2013), con mejoras significativas $\mathrm{p}<, 001$ entre los registros pre y post-test en PROM de 17,18․ Las ganancias de $17,18^{\circ}$, frente a $9,77^{\circ}$ y $13,53^{\circ}$ respectivamente con $\mathrm{HR}$ y $\mathrm{HR}+\mathrm{EE}$, se puede relacionar con un periodo mayor de entrenamiento de 9 frente a 4 semanas. También, periodos inferiores de seis semanas, indican que la ganancia mayor se produjo en las tres primeras semanas, es decir, similares a nuestro estudio (Rowlands et al., 2003). Igualmente, Kenric (2003) al comparar PNF con AIS y EEP $(n=38)$ en flexión de cadera, entrenando 4 semanas y 4 sesiones semanales, obtuvieron mejoras significativas en los tres grupos sin diferencias significativas entre ellos. Un entrenamiento realizado una vez a la semana, en un tiempo total que triplica el nuestro, han producido mejoras significativas en el ROM (Burke et al., 2001; Rowlands et al., 2003; Feland y Marín, 2004). Determinados autores postulan que una sola sesión de PNF es suficiente para incrementar el ROM (Feland, Myrer y Merrill 2001), con una mejora dependiendo de la articulación de entre 3 y 9 grados (Feland et al., 2001).

En cuanto a la contracción isométrica, gran número de estudios con PNF, utilizaron tiempos de contracción entre 3 (Bonnar, Deivert y Gould, 2004) y 15 segundos (Schuback, Hooper y Salisbury, 2004); y en la mayor parte de los estudios con un tiempo de contracción comprendido entre 3 a 15 segundos, la ganancia del ROM aumentó (Feland et al., 2001; Ferber, Osternig y Gravelle, 2002; Bonnar et al., 2004; Feland y Marín, 2004). En ciertos casos, una duración más larga de contracción isométrica está positivamente correlacionada con el aumento de ROM. Usando un procedimiento de estiramiento PNF durante 6 semanas, el cambio medio en el grupo que utilizó $5 \mathrm{~s}$ fue de $28^{\circ}$, mientras el que la mantuvo $10 \mathrm{~s}$, tuvo una ganancia ligeramente superior de $33^{\circ}$ (Rowlands et al., 2003). Otros estudios encontraron que las ganancias en ROM son independientes de la duración de la contracción isométrica (Schmitt et al., 1999; Feland et al., 2001; Bonnard et al., 2004). Estudios con tan solo 3 segundos de contracción isométrica se muestran efectivos en el tiempo (Nelson y Cornelius, 1991; Bonnard et al., 2004; González- Ravé et al., 2012).

En nuestro estudio, en ambas técnicas la contracción isométrica se realizó durante $6 \mathrm{~s}$ y hubo mejoras significativas, lo cual se encuentra dentro de los límites de la mayor parte de estudios que vieron aumentado el ROM (Schmitt et al., 1999; López-Bedoya et al., 2007; López-Bedoya et al., 2013). En ambas técnicas existen mejoras significativas en PROM entre pre y post-test, siendo ligeramente superior $\mathrm{HR}+\mathrm{EE}$. Se constata por tanto, la eficacia de la electroestimulación para la mejora del ROM como en algunos de los estudios precedentes (Pérez y Álamo, 2001; Basas, 2001; Maciel y Cámara, 2008; Espejo, Maya y Cardero, 2012; López-Bedoya et al. 2017). No obstante, aunque se trata de trabajos realizados a largo plazo como el nuestro, se han utilizado técnicas y/o programas de electroestimulación diferentes.

En el AROM, resaltar que nuestros resultados son menos favorables que en la manifestaciones pasivas. Algunos estudios (López-Bedoya et al., 2013; LópezBedoya et al., 2017), indican que las mejoras en el PROM son siempre 
superiores. Con AROM se produjeron ligeras ganancias con diferencias no significativas entre la medida pre y post-test en HR y HR+EE. Estos resultados parecen corroborar los hallados en otros estudios donde las técnicas de PNF Hold Relax, y aquellas que se caracterizan por un estiramiento pasivo, no son las más adecuadas para el desarrollo del AROM (Meroni et al., 2010; LópezBedoya et al., 2013), algo que no ocurre para el PROM.

\section{Cambios producidos en el ROM pasivo y activo entre el post y re-test}

Existen resultados contrapuestos en la revisión documental en relación a la duración de los efectos que tiene el estiramiento utilizando técnicas de PNF sobre el ROM. McCarthy, Olsen y Smeby (1997), aportaron que las ganancias en el ROM duran aproximadamente siete días después de una semana de estiramiento dos veces al día. Otros trabajos señalan que los incrementos del ROM decrecen con rapidez una vez cesa la intervención (McCarthy et al., 1997; Spernoga, Uhl, Arnold y Gansneder, 2001), aconsejándose trabajar el estiramiento mediante PNF, una o dos veces por semana para estabilizar el ROM a largo plazo, también corroborado en estudios con 3 sesiones durante 30 días (Spernoga et al., 2001).

En nuestro estudio, con la técnica HR, prácticamente no aparecen pérdidas en el PROM después de dos semanas de no entrenamiento entre el post- y re-test $\left(0,03^{\circ}\right)$, tal es así, que entre la medida pre- y re-test se mantiene una ganancia significativa de amplitud articular de 9,80 (Tabla 1). En el grupo que recibió $H R+E E$, aparecen perdidas sensiblemente superiores en los sujetos sometidos al $\mathrm{HR}+\mathrm{EE}$, entre el post- y re-test con pérdidas de 4,49․ No obstante, entre la medida de pre- y re-test, se mantiene una ganancia significativa de $9,04^{\circ}$ en el ROM.

Estos resultados constatan lo indicado por Rubley, Brucker, Ricard y Draper (2001), quienes sugieren que las ganancias obtenidas en el ROM son retenidas al menos tres semanas después del cese del programa de entrenamiento. Esto contrasta con McCarthy et al. (1997), en los que las ganancias en el ROM duran aproximadamente siete días después de una semana de estiramiento dos veces al día. Otros estudios indican que la duración de los efectos puede variar debido al tiempo de estiramiento y la duración de la contracción durante el estiramiento. Se ha demostrado que cuando la contracción se mantiene entre 3 y $10 \mathrm{~s}$ se producen mejores efectos, considerándose además como preferible un tiempo de 6 s (Rowlands et al., 2003; Feland et al., 2004).

\section{Tolerancia al estiramiento y sensación de dolor con la escala analógica digital}

En nuestro estudio, se ha querido realizar una primera aproximación que pueda aportar algunas respuestas a la incógnita subyacente en el entrenamiento de la flexibilidad y la sensación de dolor intrínseca. Resulta difícil, hacer una comparativa con otros trabajos, ya que las técnicas de FNP generalmente, se han utilizado como terapia para reducir el dolor en pacientes con síndrome de dolor miofascial, así como con patologías dolorosas en la articulación del 
hombro, obteniéndose efectos positivos según los análisis de los mismos mediante escalas analógicas digitales (Lee, Park y Na, 2013; Kim, Lee y Ha, 2015).

Únicamente Marsall et al. (2006), en cuatro semanas de estiramientos y un incremento del $20,9 \%$ en el $\mathrm{ROM}$ en isquiotibiales con straight leg raise test, midieron la intensidad del dolor provocado durante el estiramiento máximo con EVA, no encontrándose cambios significativos entre el pretest y el postest en la intensidad del dolor.

Sin embargo, en nuestro trabajo, el valor promedio de EVA, fue estadísticamente diferente entre los tratamientos $\mathrm{HR}$ y $\mathrm{HR}+\mathrm{EE}$ en la medida de la primera sesión, $p=, 003$. Lo cual sugiere niveles muy bajos de percepción del dolor en la técnica $\mathrm{HR}+\mathrm{EE}$ respecto a HR.

Sorprende que los niveles más bajos de sensación de dolor, en esta sesión, se den en el grupo $\mathrm{HR}+\mathrm{EE}$, resultado que podría deberse a la predisposición negativa hacia una técnica que incluye un elemento que se percibe como amenazante de dolor, la electroestimulación, debida a la corriente eléctrica. Esta predisposición negativa frente al dolor, puede hacer que los valores de EVA, después de experimentar realmente sobre la práctica se perciban como menos dolorosa. La tendencia a lo largo de las ocho sesiones en las que se evaluó la percepción del dolor, tiende a estabilizarse desde la primera a la cuarta sesión en niveles medios de percepción, decreciendo progresivamente desde la cuarta hasta la última sesión en la técnica $H R+E E$, y manteniéndose en niveles medios en la técnica HR, aumentando ligeramente en las dos últimas sesiones a niveles superiores sobre la técnica de HR+EE.

En nuestro estudio, al no haber diferencias significativas entre los valores promedio de EVA, según se aplicó $\mathrm{HR}$ o $\mathrm{HR}+\mathrm{EE}$, debemos descartar el efecto del dolor sobre las ganancias en PROM producidas entre pre y post-test, en sendos tratamientos. Es decir, no se obtiene más o menos ganancia porque una u otra técnica genere más o menos dolor. La puntuación media de EVA, en el total de las sesiones, fue prácticamente igual para ambos grupos con un valor de 53,28 en HR y de 50,79 en HR+EE, lo cual nos indica que ambas técnicas son bien toleradas.

No obstante, se plantea la necesidad de un mayor número de estudios, que ofrezcan respuesta a las numerosas cuestiones que son inherentes al entrenamiento de la flexibilidad y a la sensación de dolor que produce.

\section{CONCLUSIONES}

El entrenamiento de la flexibilidad basado en técnicas de PNF Hold Relax y PNF Hold Relax con contracción isométrica inducida mediante electroestimulación, incrementan el ROM significativamente en su manifestación pasiva, sin diferencias significativas entre ambas.

La mejora del ROM en su manifestación activa no se ha visto incrementada 
significativamente en ninguno de los grupos, lo cual nos induce a pensar que dichas técnicas no se adaptan adecuadamente a dicho objetivo.

En relación al periodo post entrenamiento, no existen pérdidas significativas del rango de movimiento en su manifestación pasiva.

La aplicación de las técnicas de HR y HR+EE generan valores promedios similares en la escala EVA, siendo bien toleradas en cuanto a la percepción del dolor.

\section{Aplicaciones prácticas}

Como consecuencia de las conclusiones obtenidas, se podría considerar que el entrenamiento de la flexibilidad en deportistas y jóvenes adultos mediante técnicas de FNP que incluyen un estiramiento pasivo, tipo HR con y sin contracción isométrica inducida mediante electroestimulación son indicadas para la mejora del PROM, no siendo así, para el ROM activo.

Por tanto, el uso de estas técnicas en deportes gimnásticos, especialmente en gimnasia artística, rítmica y acrobática, que requieren altos niveles de ROM pasivo para ejecutar posiciones estáticas específicas con la máxima calidad técnica son adecuadas (Harvey y Mansfield, 2000; Sands, McNeal, Stone, Russell y Jemni, 2006; Douda, Toubekis, Avloniti y Tokmakidis, 2008). No obstante, para la mejora del AROM, variable también destacable en estas disciplinas gimnásticas, así como en deportes de lucha, patinaje artístico, natación sincronizada, entre otros, sería aconsejable otras técnicas de FNP tipo CRAC, debido al mecanismo de inervación recíproca, contrastada en diferentes estudios (Sharman et al., 2006; López Bedoya et al., 2007).

\section{REFERENCIAS BIBLIOGRÁFICAS}

Adler, S. S.; Berkers, D. \& Buck, M. (2002). La Facilitación Neuromuscular Propioceptiva en la práctica. Guía ilustrada. Buenos Aires: Editorial Panamericana.

Basas, A. (2001). Metodología de la electroestimulación en el deporte. Revista Fisioterapia, 23, 36-47. https://doi.org/10.1016/S0211-5638(01)72971-9

Bernhart, C. M. (2013). A Review of Stretching Techniques and Their Effects on Exercise. Senior Honors Theses. Available from: http://digitalcommons.liberty.ed/honors/383.

Besson, J.M. \& Chaouch, A. (1987). Peripheral and spinal mechanisms of nocipception. Physiological Reviews, 67, 67-186. https://doi.org/10.1152/physrev.1987.67.1.67

Bonnar, B. P., Deivert, R. G. \& Gould, T. E. (2004). The relationship between isometric contraction durations during hold-relax stretching and improvement of hamstring flexibility. The Journal of Sports Medicine and Physical Fitness, 44, 258-261.

Burke, D. G., Holt, R., Rasmussen, N. C., MacKinnon, J. F., Vossen y Pelham, T. W. (2001). Effects of hot or cold water immersion and 
modified proprioceptive neuromuscular facilitation flexibility exercises on hamstring length. J. Athletic Train, 36, 16-19.

Douda, H. T., Toubekis, A. G, Avloniti, A. A. \& Tokmakidis, S. P. (2008). Physiological and Anthropometric Determinants of Rhythmic Gymnastics Performance. International Journal of Sports Physiology and Performance, 3, 41-54. https://doi.org/10.1123/ijspp.3.1.41

Espejo, L., Maya, J., Cardero, M. A., \& Albornoz, M. (2012). Aumento de la extensibilidad isquiotibial tras aplicar elongación muscular eléctrica. Fisioterapia. 34(3), 112-117. https://doi.org/10.1016/i.ft.2012.01.001

Feland, J. B., \& Marin, H. N. (2004). Effect of submaximal contraction intensity in contract-relax proprioceptive neuromuscular facilitation stretching. British Journal of Sports Medicine, 38(4): e18. https://doi.org/10.1136/bjsm.2003.010967

Feland J. B., Myrer J. W., Merrill R. M. (2001). Acute changes in hamstring flexibility: PNF versus static stretch in senior athletes. Physical Therapy in Sport, 2(4), 186-193. https://doi.org/10.1054/ptsp.2001.0076

Ferber, R., Osternig, L., Gravelle, D. (2002). Effect of PNF stretch techniques on knee flexor muscle EMG activity in older adults. Journal of Electromyography and Kinesiology, 12(5), 391-397. https://doi.org/10.1016/S1050-6411(02)00047-0

Funk, D. C., Swank, A. M., Mikla, B. M., Fagan, T. A., \& Farr, B. K. (2003). Impact of prior exercise on hamstring flexibility: a comparison of proprioceptive neuromuscular facilitation and static stretching. Journal of Strength and Conditioning Research, 17(3), 489-492. https://doi.org/10.1519/00124278-200308000-00010

García-Manso, J. M., López-Bedoya, J., Rodríguez-Matoso, D., Ariza-Vargas, L., Rodríguez-Ruiz, D. \& Vernetta, M. (2015). Static-stretching vs. contract-relax - proprioceptive neuromuscular facilitation stretching: study the effect on muscle response using tensiomyography. European Journal of Human Movement, 34, 96-108.

González-Ravé, J, M., Sánchez-Gómez, A. \& Santos-García, D. J. (2012). Efficacy of Two Different Stretch Training Programs (Passive vs. Proprioceptive Neuromuscular Facilitation) on Shoulder and Hip Range of Motion in Older People. Journal of Strength \& Conditioning Research, 26(4), 1045-1051.

https://doi.org/10.1519/JSC.0b013e31822dd4dd

Harvey, D. \& Mansfield, C. (2000) Measuring Flexibility for Performance and Injury Prevention. En Gore (ed) Physiological Tests for Elite Athletes (pp. 98-113). Australian Sports Commission. Champaign: Human Kinetics.

Holt, L. E., Holt, J. B., Pelham, T. W. (1996). What research tells us about flexibility, I. Biomechanics in Sports. 13, 175-179.

Kenric, L. (2003). A Comparison of Different Methods for Improving Hamstring Flexibility. Tesis Doctoral. Florida Atlantic University.

Kim, J. J., Lee, S. Y. \& Ha, K. (2015). The efects of exercise using PNF in patients with a supraspinatus muscle tear. Journal of Physical Therapy Science, 27(8), 2443-2446. 
https://doi.org/10.1589/jpts.27.2443

Lee, J. H., Park, S. J., Na, S. S. (2013). The Effect of Proprioceptive Neuromuscular Facilitation Therapy on Pain and Function. Journal of Physical Therapy Science, 25, 713-716. https://doi.org/10.1589/jpts.25.713

Lopez-Bedoya, J., Robles, A., Vernetta, M., Piedra, J. \& Nunez, J. J. (2007). Efecto de dos tipos de entrenamiento de flexibilidad, hold relax (HR) y active isolated stretching (AIS), sobre el rango de movimiento pasivo de cadera en abduccion. En: ACUGA \& Alto Rendimiento (eds.) CD de actas del III Congreso Nacional de Ciencias del Deporte: Nutricion, Medicina y Rendimiento en el Joven Deportista. Pontevedra: Editores.

López-Bedoya, J., Vernetta, M., Lizaur, P., Martínez-Patiño, M. J. \& Ariza-Vargas, L. (en prensa). Comparación de técnicas de entrenamiento de flexibilidad (FNP) con y sin electroestimulación. Revista Internacional de Medicina y Ciencias de la Actividad Física y el Deporte.

López-Bedoya, J., Vernetta, M., Robles, A \& Ariza-Vargas, L. (2013). Effect of three types of flexibility training on active and passive hip range of motion. The Journal of Sports Medicine and Physical Fitness, 53(3), 304-11.

Maciel, A. C. \& Câmara, S. M. (2008). Influência da estimulação elétrica nervosa transcutânea (TENS) associada ao19.alongamento muscular no ganho de flexibilidade. Revista Brasileira de Fisioterapia, 5(12), 373-378. https://doi.org/10.1590/S1413-35552008000500006

Merskey, H. \& Bogduk, N. (2005). Classification of Chronic Pain. Descriptions of Chronic Pain Syndromes and Definitions of Pain Terms. Annals of Neurology, 57(3), 425-429.

Marshall, P. W., Cashman, A. \& Cheema, B. S. (2006). A randomized controlled trial for the effect of passive stretching on measures of hamstring extensibility, passive stiffness, strength, and stretch tolerance. Journal of Science and Medicine in Sport, 14(6), 535-540. https://doi.org/10.1016/j.jsams.2011.05.003

McCarthy P. W., Olsen J. P. \& Smeby I. H. (1997). Effects of contract-relax stretching procedures on active range of motion of the cervical spine in the transverse plane. Clinical Biomechanics, 12(2), 136-138.

https://doi.org/10.1016/S0268-0033(96)00060-5

Meroni, R., Cerri, C. G., Lanzarini, C., Barindelli, G., Della Morte, G., Gessaga, V., ... \& De Vito, G. (2010). Comparison of active stretching technique and static stretching technique on hamstring flexibility. Clinical journal of sport medicine, 20(1), 8-14. https://doi.org/10.1097/JSM.0b013e3181c96722

Nelson K. C. \& Cornelius W. L. (1991).The relationship between isometric contraction durations and improvement in shoulder joint range of motion. The Journal of Sports Medicine and Physical Fitness, 31(3), 385-388

Olesen, A.E., Andresen, T., Staahl, C. \& Drewes, A.M. (2012). Human experimental pain models for assessing the therapeutic efficacy of analgesic drugs. Pharmacological reviews, 64, 722-79. https://doi.org/10.1124/pr.111.005447

Page, P. (2012). Current concepts in muscle stretching for exercise and rehabilitation. The International Journal of Sports Physical Therapy, 7(1), 109-119. 
Pérez, M. J. \& Álamo, A. D. (2001). Comparative study between muscular stretching by active tension and electrostimulation. Fisioterapia, 23, 10-14. https://doi.org/10.1016/S0211-5638(01)72924-0

Prentice, WE (1994). Therapeutic Modalities in Sports Medicine. London: Mosby.

Rowlands, A. V., Marginson, V. \& Lee, J. (2003). Chronic flexibility gains: effect of isometric contraction duration during proprioceptive neuromuscular facilitation stretching techniques. Research Quarterly for Exercise and Sport, 74(1), 47-52. https://doi.org/10.1080/02701367.2003.10609063

Rubley, M. D., Brucker, J. B., Ricard, M. D. \& Draper, D. O. (2001). Flexibility retention 3 weeks after a 5-day training regime. Journal of Sport Rehabilitation, 10, 105-112.

https://doi.org/10.1123/isr.10.2.105

Sands, W. A., McNeal, J. R., Stone, M. H., Russell, E. M., \& Jemni, M. (2006). Flexibility enhancement with vibration: Acute and long-term. Medicine \& Science in Sports \& Exercise, 38(4), 720-725. https://doi.org/10.1249/01.mss.0000210204.10200.dc

Sands, W. A., McNeal, J. R., Stone, M. H., Haff, G. G. \& Kinser, M. (2008). Effect of Vibration on Forward Split Flexibility and Pain Perception in Young Male Gymnasts. International Journal of Sports Physiology and Performance, 3, 469-481. https://doi.org/10.1123/ijspp.3.4.469

Schmitt, G. D., Pelham, T. W., \& Holt, L. E. (1999). Comparison of selected protocols during propioceptive neuromuscular facilitation stretching. Clinical Kinesiology, 53(1), 16-21. Schuback, B., Hooper, J., Salisbury, L. (2004). A comparison of a self- stretch incorporating proprioceptive neuromuscular facilitation components and a therapist-applied PNF-technique on hamstring flexibility. Physiotherapy, 90(3), 151-157.

https://doi.org/10.1016/i.physio.2004.02.009

Sharman, M. J., Cresswel, A. G. \& Riek, S. (2006). Proprioceptive Neuromuscular Facilitation Stretching Mechanisms and Clinical Implications. Sports Medicine, 36(11), 929-939.

https://doi.org/10.2165/00007256-200636110-00002

Spernoga, S. G., Uhl, T. L., Arnold, B. L., \& Gansneder, B. M. (2001). Duration of maintained hamstring flexibility after a one-time, modified hold-relax stretching protocol. Journal of athletic training, 36(1), 44.

Sundquist, R. D. (1996). The comparative effectiveness of static stretching and propioceptive neuromuscular facilitation stretching techniques in increasing hip flexion range of motion. Microform publications, for Sport \& Human Performance, Universidad de Oregon.

Surburg, P. R. \& Schrader, J. W. (1997). Proprioceptive Neuromuscular Facilitation Techniques in Sports Medicine: A Reassessment. Journal of Athletic Training, 31(1), 34-39.

Voss, D. E., Ionta, M. K. \& Meyers, B. J. (2004). Facilitación Neuromuscular Propioceptiva. Patrones y técnicas. Buenos Aires: Editorial Panamericana.

Zajac, A. \& Nowak, K. (1997). Flexibility and different training methods. Wychowanie- fizyczne- sport- Warsaw, 41(3), 37-45. 
Rev.int.med.cienc.act.fís.deporte - vol. 20 - número 80 - ISSN: 1577-0354

Número de citas totales / Total references: 42 (97,6\%)

Número de citas propias de la revista / Journal's own references: 1 (2,3\%)

Rev.int.med.cienc.act.fís.deporte - vol. 20 - número 80 - ISSN: 1577-0354 